\title{
A NOTE FROM THE PUBLISHER: FIGURES AND ILLUSTRATIONS
}

About $90 \%$ of the figures and illustrations in our journals fit into a single column which is $80 \mathrm{~mm}$ wide. Almost always-as they say in fuzzy set language-the size of the submitted figure must be reduced. If all such figures are properly prepared, so that they can be reduced to $40 \%$ of the submitted size, then we reduce printing costs and publication lag and improve aesthetic quality. And this can be done with very little extra effort and no added cost to authors. Therefore, authors are requested to comply with these guidelines for figures suitable for a single column.

(1) The width of the submitted figure is extremely important. To fit an 80-mm column, the submitted size cannot exceed $200 \mathrm{~mm}$. But any size between 175 and $200 \mathrm{~mm}$ will result in an attractive figure. Therefore, submit figures that are between 175 and $200 \mathrm{~mm}$ wide. (We will, of course, always be delighted to accept figures that are already reduced to a width of $70-80 \mathrm{~mm}$ if they are on positive stat.)

(2) Use letters and numbers not less than 12 points $(3 \mathrm{~mm})$ or more than 24 points $(6.5 \mathrm{~mm})$ in size.

12-point

$100 \%$

$40 \%$

\section{2-point}

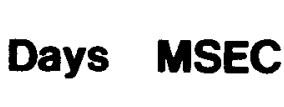

Dop MSEC 24-point

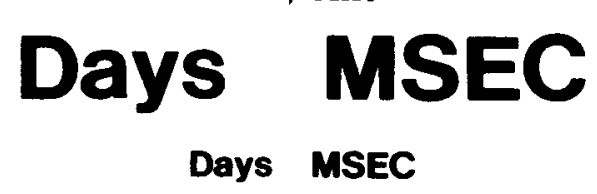

(3) Use Helvetica. Trade Gothic, or similar type style of medium weight (thickness), that is, not bold or light. Lines and symbols should be proportionately the same weight. (If lines or strokes of letters are too thin, they tend to disappear in reduction. If they are too thick, individual letters lose distinctiveness, i.e., "a" or "e" becomes a dot.)

(4) Bear in mind that spaces between lines and letters are also reduced, so make such separations wide enough so that plot lines will not lose their separations and words will not become illegible.

(5) Open circles should be drawn about twice the diameter of closed circles so that they will not close up in reduction.

Graphic aids such as Chartpak, Formatt, or Leroy lettering sets are fine for graphs and line drawings, but all work must be opaque and neat. We are not equipped to do any art work or touch up. Only one of each figure need be of professional quality (the others can be Xeroxed) and that can be the original, a PMT stat, or other photographic reproduction. If the original is submitted, it should be on a good grade of bond, blue-lined grid paper, or matte acetate. For mailing, original figures should be protected between two sheets of cardboard.

Figures that require a double column are, of course, acceptable and will receive individual treatment. Photographs involving halftones should be given special attention as to detail and figure-ground contrast.

A list of figure captions should be included on a separate sheet, that is, the caption should not appear on the figure itself. 